\title{
Origins of oscillatory dynamics in the model of reactive oxygen species in the rhizosphere
}

\author{
Stevan Maćešić, ${ }^{1,}$ a) Ágota Tóth, ${ }^{1}$ and Dezső Horváth ${ }^{2}$, b) \\ 1) Department of Physical Chemistry and Materials Science, University of Szeged, Rerrich Béla tér 1, 6720 Szeged, \\ Hungary \\ ${ }^{2)}$ Department of Applied and Environmental Chemistry, University of Szeged, Rerrich Béla tér 1, 6720 Szeged, \\ Hungary
}

(Dated: 21 September 2021)

\begin{abstract}
Oscillatory processes are essential for normal functioning and survival of biological systems and reactive oxygen species have a prominent role in many of them. A mechanism representing the dynamics of these species in the rhizosphere is analyzed using stoichiometric network analysis with the aim to determine its capabilities to simulate various dynamical states including oscillations. A detailed analysis has shown that unstable steady states result from four destabilizing feedback cycles, among which the cycle involving hydroquinone, an electron acceptor and its semi-reduced form, is the dominant one responsible for the existence of saddle-node and Andronov-Hopf bifurcations. This requires higher steady-state concentration for the reduced electron acceptor compared to that of the remaining species, where the level of oxygen steady-state concentration determines whether Andronov-Hopf or saddle-node bifurcation will occur.
\end{abstract}

\section{INTRODUCTION}

Various systems in chemistry and biology ${ }^{1}$ are known for the ability of self-organization when found under conditions far from equilibrium. As a result of this, they are capable of exhibiting different types of temporal and spatio-temporal phenomena such as bistability ${ }^{2-4}$, oscillations $^{5-8}$, $\operatorname{chaos}^{9,10}$, reaction-diffusion fronts ${ }^{7,11-13}$, etc. Oscillatory dynamics is especially important for biological systems where many processes are characterized by periodic behavior with periods ranging from seconds (calcium oscillations) ${ }^{14,15}$, minutes (glycolytic oscillations) $)^{16,17}$ to the hours (circadian clock) ${ }^{18}$, days and months (hormonal oscillations) ${ }^{19-21}$. For survival, biological systems have to adapt to sudden and very often periodical changes in their surroundings. Hence many biological processes are also periodic, therefore understanding their mechanisms is of great importance.

Reactive oxygen species (ROS) are closely related to the oscillatory dynamics of biological processes. ${ }^{22}$ ROS are byproducts of biochemical reactions that involve $\mathrm{O}_{2}$ and produce energy vital for the normal functioning of plants and other aerobic organisms. ROS are formed as a result of the ability of $\mathrm{O}_{2}$ to easily accept electrons because of the two unpaired electrons in the outermost orbital. The presence of ROS can have negative and positive impacts on the biological systems depending on the level of their concentrations. ROS have an important role as signaling molecules in plants when they are present in low concentrations ${ }^{23,24}$, but high ROS concentrations can make irreparable damage due to their high reactivity which allows them to oxidize almost all biological molecules, including DNA, proteins and lipids ${ }^{25-28}$.

a) Also at Faculty of Physical Chemistry, University of Belgrade, Studentski Trg 12, 11158 Belgrade, Serbia

b) Electronic mail: horvathd@chem.u-szeged.hu
Taran et al. ${ }^{29}$ conducted a series of experiments to investigate ROS dynamics in the rhizosphere, the narrow zone of soil along plant root surfaces. In this area, ROS are produced as a result of various redox processes. A model system was designed mimicking the rizosphere in which they focused on reactions between two redox pairs, 2,6-dimethoxybenzoquinone/hydroquinone and methylene blue/leuco-methylene blue, in the presence of both sodium borohydride, as a reducing agent, and oxygen. Experiments were carried out both in wellstirred reactors and in thin layers of solution where diffusion is present. They have found that the system can display autocatalytic behavior and produce reactiondiffusion fronts. Based on these results they proposed a reaction network that resembles the mechanism of the reaction between methylene blue and sulfide ion, which is known to produce oscillations. ${ }^{30}$

In this work we carry out a thorough investigation of the network that is the core of their proposed model to analyze its full capacity to produce oscillatory dynamics and to determine the conditions required for its existence. By performing stability analysis using stoichiometric network analysis (SNA) ${ }^{31-34}$, we will show that destabilizing feedback cycles can be detected and we will identify the conditions essential for the emergence of bistability and oscillations.

\section{MODEL AND METHODS}

\section{A. Model}

The model, defined by reactions in eqn (R1-R9), is represented in Fig. 1. Quinone is reduced by sodium borohydride in the first step (R1). Synproportionation of hydroquinone and quinone takes place according to eqn (R2) forming the semiquinone radical that can react with oxygen producing a reactive oxygen species in eqn 
(R3). In a similar fashion, synproportionation of the second redox pair, methylene blue and leucomethylene blue in this specific case, in eqn (R4) gives a reactive intermediate $\mathrm{MB}^{\circ}$ radical, which reacts with dissolved oxygen in eqn (R5) producing a reactive oxygen species. The coupling between the methylene blue and hydroquinone systems is via a two-electron redox process in eqn (R6). The disproportionation of ROS to oxygen and hydrogen peroxide is according to in eqn (R7). The dissolution of oxygen follows eqn (R8), while the decomposition of sodium borohydride eqn (R9).

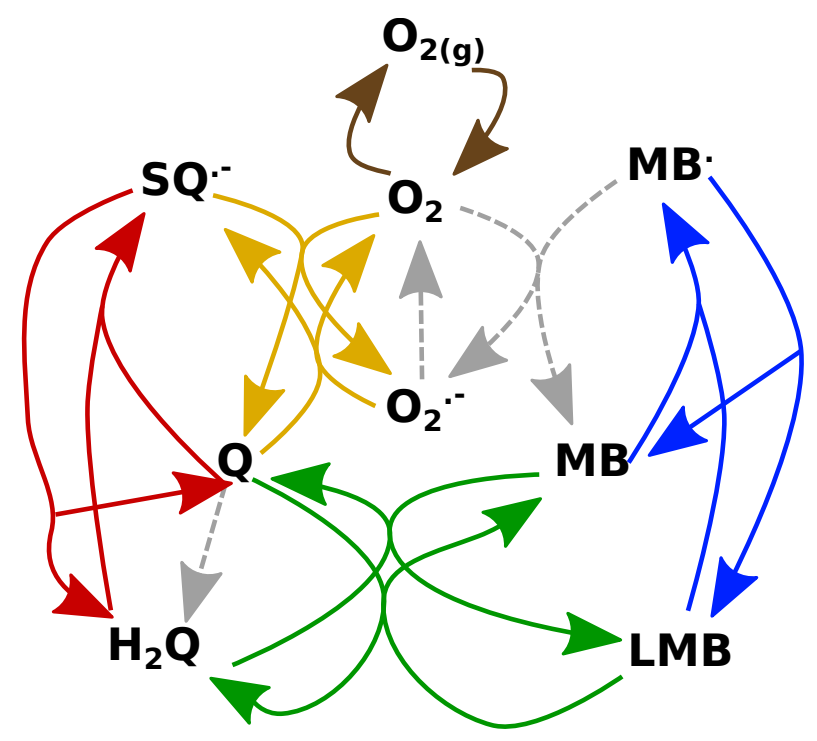

FIG. 1. Schematic representation of the model (R1)-(R9) and the corresponding reaction rates presented in Table I. The colored solid lines show the reversible reactions only.

$$
\begin{aligned}
\mathrm{NaBH}_{4}+\mathrm{Q} & \rightarrow \text { borates }+\mathrm{H}_{2} \mathrm{Q} \\
\mathrm{H}_{2} \mathrm{Q}+\mathrm{Q} & \rightleftharpoons 2 \mathrm{SQ}^{--}+2 \mathrm{H}^{+} \\
\mathrm{SQ}^{--}+\mathrm{O}_{2} & \rightleftharpoons \mathrm{Q}+\mathrm{O}_{2}^{--} \\
\mathrm{MB}+\mathrm{LMB} & \rightleftharpoons 2 \mathrm{MB}^{\cdot}+2 \mathrm{H}^{+} \\
\mathrm{MB}+\mathrm{O}_{2} & \rightarrow \mathrm{MB}+\mathrm{O}_{2}^{--} \\
\mathrm{MB}+\mathrm{H}_{2} \mathrm{Q} & \rightleftharpoons \mathrm{LMB}^{-} \mathrm{Q} \\
2 \mathrm{O}_{2}^{--}+2 \mathrm{H}^{+} & \rightleftharpoons 2 \mathrm{HO}_{2} \rightarrow \mathrm{O}_{2}+\mathrm{H}_{2} \mathrm{O}_{2} \\
\mathrm{O}_{2}(\mathrm{~g}) & \rightleftharpoons \mathrm{O}_{2} \\
\mathrm{NaBH}_{4} & \rightarrow \text { borates }+\mathrm{H}_{2}
\end{aligned}
$$

In this complex reaction network the concentrations of eight species, 2,6-dimethoxybenzoquinone (Q), hydroquinone $\left(\mathrm{H}_{2} \mathrm{Q}\right)$, semiquinone $\left(\mathrm{SQ}^{--}\right)$, oxygen $\left(\mathrm{O}_{2}\right)$, superoxide radical $\left(\mathrm{O}_{2}^{--}\right)$, methylene-blue $(\mathrm{MB})$, leucomethylene blue (LMB) and semi-reduced methylene blue $\left(\mathrm{MB}^{*}\right)$, comprise the dynamical variables. The main reactant, sodium borohydride, is applied in a concentration considerably higher than the concentrations of other
TABLE I. Rate equations of the network in Fig. 1. with rate coefficients allowing oscillatory dynamics.

$$
\begin{array}{l|l}
r_{1}=k_{1}^{\prime}[\mathrm{Q}] & k_{1}^{\prime}=1.00 \times 10^{-2} \mathrm{~s}^{-1} \\
r_{2}=k_{2}\left[\mathrm{H}_{2} \mathrm{Q}\right][\mathrm{Q}] & k_{2}=1.00 \times 10^{3} \mathrm{M}^{-1} \mathrm{~s}^{-1} \\
\left.r_{-2}=k_{-2}[\mathrm{SQ}]^{-}\right]^{2} & k_{-2}=1.00 \times 10^{8} \mathrm{M}^{-1} \mathrm{~s}^{-1} \\
r_{3}=k_{3}[\mathrm{SQ} \cdot-]\left[\mathrm{O}_{2}\right] & k_{3}=7.35 \times 10^{4} \mathrm{M}^{-1} \mathrm{~s}^{-1} \\
r_{-3}=k_{-3}[\mathrm{Q}]\left[\mathrm{O}_{2}^{-}\right] & k_{-3}=4.93 \times 10^{3} \mathrm{M}^{-1} \mathrm{~s}^{-1} \\
r_{4}=k_{4}[\mathrm{MB}][\mathrm{LMB}] & k_{4}=6.47 \times 10^{3} \mathrm{M}^{-1} \mathrm{~s}^{-1} \\
r_{-4}=k_{-4}[\mathrm{MB} \cdot]^{2} & k_{-4}=1.00 \times 10^{8} \mathrm{M}^{-1} \mathrm{~s}^{-1} \\
r_{5}=k_{5}[\mathrm{MB}]\left[\mathrm{O}_{2}\right] & k_{5}=1.38 \times 10^{5} \mathrm{M}^{-1} \mathrm{~s}^{-1} \\
r_{6}=k_{6}[\mathrm{MB}]\left[\mathrm{H}_{2} \mathrm{Q}\right] & k_{6}=2.85 \times 10^{4} \mathrm{M}^{-1} \mathrm{~s}^{-1} \\
r_{-6}=k_{-6}[\mathrm{LMB}][\mathrm{Q}] & k_{-6}=1.25 \times 10^{2} \mathrm{M}^{-1} \mathrm{~s}^{-1} \\
r_{7}=k_{7}\left[\mathrm{O}_{2}^{--}\right]^{2} & k_{7}=1.80 \times 10^{4} \mathrm{M}^{-1} \mathrm{~s}^{-1} \\
r_{8}=k_{8}^{\prime} & k_{8}^{\prime}=2.25 \times 10^{-7} \mathrm{M} \mathrm{s}^{-1} \\
r_{-8}=k_{-8}\left[\mathrm{O}_{2}\right] & k_{-8}=1.00 \times 10^{-3} \mathrm{~s}^{-1}
\end{array}
$$

species and therefore it is considered as a pool chemical with a constant concentration. Even though we drive the reduction process with the excess of borohydride, in the presence of $\mathrm{O}_{2}$ in the solution, ROS are formed by two redox cycles that are mutually coupled.

In our analysis we consider pseudo steady states with borohydride in great excess, therefore, without the loss of generality, reaction $\left(R_{9}\right)$ is omitted from the model. This condition is important because the hydrolysis of sodium borohydride itself can follow oscillatory dynamics in a continuously stirred tank reactor ${ }^{35,36}$. Here we focus on the dynamics of the redox couples which requires the reducing agent in great excess. The concentration of $\mathrm{H}^{+}$ is considered to be constant at the level of $\mathrm{pH}=8.5$ and it is included in the values of the appropriate rate constants. ${ }^{29}$ The constant $\mathrm{pH}$ can only be maintained with a buffer system that is chemically inert with respect to reactions (R1)-(R9), which is indeed the case in the rhizosphere. Reaction $\left(R_{7}\right)$ involves a fast preequilibrium that transforms $\mathrm{O}_{2}^{--}$and $\mathrm{H}^{+}$directly into $\mathrm{O}_{2}$ and $\mathrm{H}_{2} \mathrm{O}_{2}$. The oxygen level in the air is considered constant, hence a constant rate for the dissolution is set in accordance with the solubility of molecular oxygen. One set of parameters used in the simulations is listed in Table I.

\section{B. Methods}

Numerical simulations and stoichiometric network analysis were performed using the program Octave, where the solutions of algebraic equations provide the steady states and the stability if needed as a function of the parameters. For solving the ordinary differential equations in order to describe the temporal evolution of the species, LSODE function with a backward differentiation formula as the integration method was utilized with the absolute tolerance set to $10^{-14}$. 


\section{RESULTS AND DISCUSSION}

\section{Stoichiometric network analysis}

The essence of stoichiometric network analysis (SNA) is the handling of reaction rates as variables instead of the concentrations used in the classical approach. ${ }^{37-39}$ This facilitates the selection of reactions that are essential in the appearance of instabilities and allows the reduction in the number of parameters. Hence with this powerful method, we can identify the sub-networks that carry the instability that can lead to oscillations.

In the first step, the steady-state equations are expressed as a linear combination of the reaction rates $\mathbf{r}_{s s}$ as

$$
\frac{d \mathbf{c}}{d t}=\mathbf{f}=\mathbf{S} \mathbf{r}_{s s}=0
$$

where $\mathbf{S}$ represents the stoichiometric matrix of the dynamical variables. In our model the rank of the stoichiometric matrix $\mathbf{S}$ is 6 , while there are 8 dynamical variables. This is a direct consequence of the two conservation constraints from the stoichiometry of quinone and the methylene blue species as

$$
\begin{gathered}
{[\mathrm{Q}]+\left[\mathrm{H}_{2} \mathrm{Q}\right]+\left[\mathrm{SQ}^{\cdot-}\right]=[\mathrm{Q}]_{0}+\left[\mathrm{H}_{2} \mathrm{Q}\right]_{0}+\left[\mathrm{SQ}^{\cdot-}\right]_{0}=Q_{T}(2)} \\
{[\mathrm{MB}]+[\mathrm{LMB}]+\left[\mathrm{MB}^{*}\right]=[\mathrm{MB}]_{0}+[\mathrm{LMB}]_{0}+\left[\mathrm{MB}^{*}\right]_{0}=M B_{T}}
\end{gathered}
$$

where $\mathrm{T}$ subscript refers to the total amount of quinone and methylene blue and 0 subscript to the initial values.

The solutions of eqn (1) ${ }^{40-42}$ for the rates yields the matrix of extreme currents $\mathbf{E}^{43}$

$$
\begin{aligned}
& \begin{array}{llllllll}
E_{1} & E_{2} & E_{3} & E_{4} & E_{5} & E_{6} & E_{7} & E_{8}
\end{array}
\end{aligned}
$$

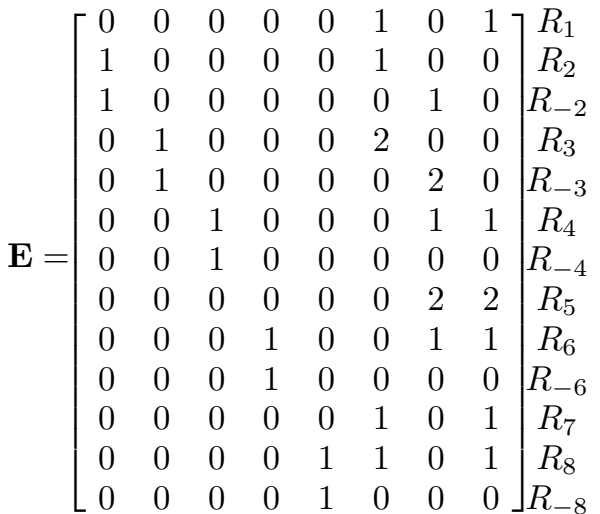

In matrix $\mathbf{E}$ each column represents one reaction route in the steady-state (also termed a sub-network capable to achieve a steady state), while each row contains the contribution of a chemical reaction to the relevant subnetwork. All elements of matrix $\mathbf{E}$ are non-negative numbers and each direction in the reversible reactions is considered as a separate reaction. In this way the governing equations at the steady state $r_{i, s s}$ can be expressed as positive linear combination of the columns of matrix $\mathbf{E}$ by introducing new parameters, i.e., current rates $\mathbf{j}$ and by using the main relation of SNA $\mathbf{r}_{s s}=\mathbf{E} \mathbf{j}$. Current rates $j_{i}$ are non-negative numbers that represent the contribution of each reaction route $E_{i}$ to the corresponding reaction rate in a steady state.

Matrix $\mathbf{E}$ of our model has 8 columns, i.e., 8 steadystate reaction routes. Among them 5 are equilibrium ones consisting of only reversible reactions with no net reaction: $E_{1}\left(R_{2} / R_{-2}\right), E_{2}\left(R_{3} / R_{-3}\right), E_{3}\left(R_{4} / R_{-4}\right)$, $E_{4}\left(R_{6} / R_{-6}\right)$ and $E_{5}\left(R_{8} / R_{-8}\right)$. Alone, they cannot produce instabilities, but in combination with other reaction routes they can significantly contribute to the rise of instabilities. Reaction route $E_{6}\left(R_{1}, R_{2}, R_{3}, R_{7}, R_{8}\right)$ (see Fig. 2) includes only reactions which describe $\mathrm{Q}$ redox cycle coupled with self-oxidation of $\mathrm{O}_{2}$. Reaction routes $E_{7}\left(R_{-2}, R_{-3}, R_{4}, R_{5}, R_{6}\right)$ and $E_{8}\left(R_{1}, R_{4}, R_{5}, R_{6}, R_{7}, R_{8}\right)$ (see Fig. 2) are minimal sub-networks that include coupling between $\mathrm{Q}$ and $\mathrm{MB}$ redox cycles. In the case of sub-network $E_{7}$, this coupling is done in two ways to maintain the steady-state: directly through reaction $R_{6}$ and indirectly by species $\mathrm{O}_{2}$ and $\mathrm{O}_{2}^{--}$. Contrarily, in subnetwork $E_{8}$ coupling exists only directly through reaction $R_{6}$, while $\mathrm{O}_{2}$ maintains the $\mathrm{MB}$ redox cycle. Reaction route $E_{7}$ has no net reaction, while $E_{6}$ and $E_{8}$ share the common net reaction of

$$
\mathrm{NaBH}_{4}+\mathrm{O}_{2}(\mathrm{~g}) \rightarrow \text { borates }+\mathrm{H}_{2} \mathrm{O}_{2}
$$
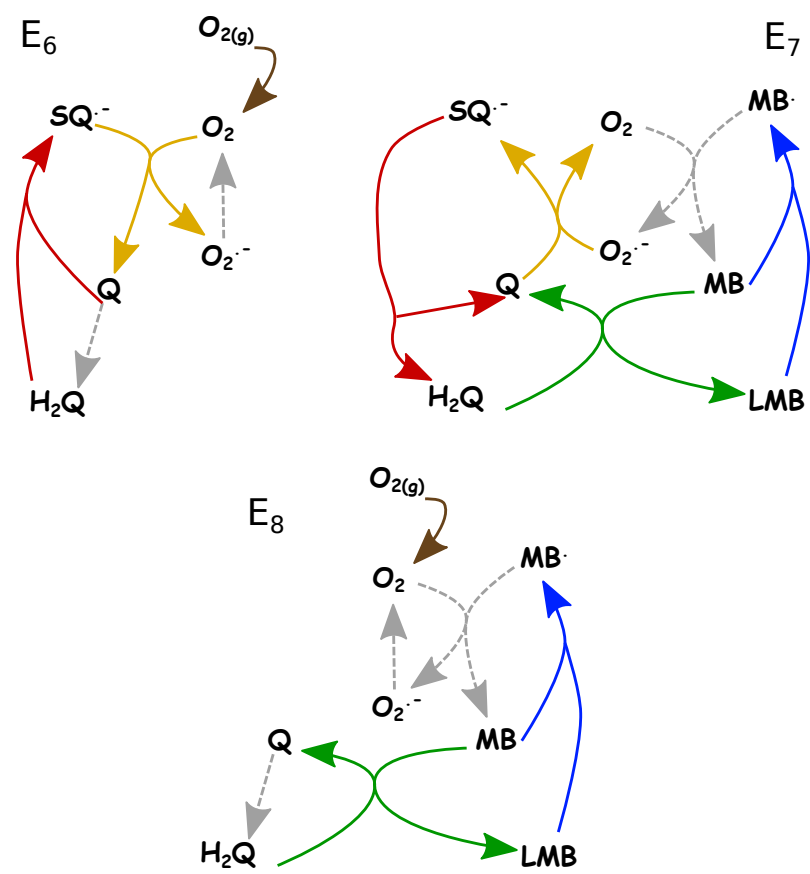

FIG. 2. Schematic representation of reaction routes: $E_{6}, E_{7}$, $E_{8}$

Using the equation $\mathbf{r}_{s s}=\mathbf{E} \mathbf{j}$, the reaction rates in the steady states can be expressed as the following linear 
combinations of $j_{i}$

$$
\begin{array}{ll}
r_{1, s s}=j_{6}+j_{8} & \\
r_{2, s s}=j_{1}+j_{6} & r_{-2, s s}=j_{1}+j_{7} \\
r_{3, s s}=j_{2}+2 j_{6} & r_{-3, s s}=j_{2}+2 j_{7} \\
r_{4, s s}=j_{3}+j_{7}+j_{8} & r_{-4, s s}=j_{3} \\
r_{5, s s}=2 j_{7}+2 j_{8} & \\
r_{6, s s}=j_{4}+j_{7}+j_{8} & r_{-6, s s}=j_{4} \\
r_{7, s s}=j_{6}+j_{8} & \\
r_{8, s s}=j_{5}+j_{6}+j_{8} & r_{-8, s s}=j_{5}
\end{array}
$$

The stability of the steady-state can be determined by calculating the eigenvalues of the Jacobian matrix (M) that contains the $\partial f_{i} / \partial c_{j}$ terms evaluated at the steady state. In $\mathrm{SNA}^{31,43,44}$ it is expressed as a function of rates according to

$$
\mathbf{M}(\mathbf{j}, \mathbf{h})=\mathbf{S} \operatorname{diag}(\mathbf{E} \mathbf{j}) \mathbf{K}^{T} \operatorname{diag}(\mathbf{h})
$$

where $\mathbf{K}$ is the matrix of reaction orders, and $\mathbf{h}=1 / \mathbf{c}_{s s}$ represents the vector of the reciprocal steady state concentrations. Equation (6) results from elementary steps in the model, for which $\partial r_{i} / \partial c_{j}=K_{i, j} r_{i} / c_{j}$ is valid. When one or more eigenvalues have positive real parts, the steady state is unstable, otherwise it is stable.

An efficient way to determine the stability is to use the so-called Hurwitz determinants or the $\alpha$-approximation, according to which an eigenvalue with a positive real part occurs when some coefficient $\alpha_{i}$ of the characteristic polynomial is negative. ${ }^{44,45}$ Condition for the appearance of Andronov-Hopf (AH) bifurcation is that the Hurwitz determinant $\Delta_{n-1}=0^{45}$, while the condition for the appearance of saddle-node ( $\mathrm{SN}$ ) bifurcation is that $\alpha_{n}=$ $0^{44}$, where $n$ is the number of linearly independent dynamical variables.

In the case of complex models the search for conditions supporting instability becomes simpler if we inspect the matrix of current rates $\mathbf{V}(\mathbf{j})$, defined as the $\mathbf{j}$-dependent part of eqn (6) according to

$$
\mathbf{V}(\mathbf{j})=-\mathbf{S} \operatorname{diag}(\mathbf{E} \mathbf{j}) \mathbf{K}^{T}
$$

Instability can now be detected to a good approximation $^{31}$ by the existence of at least one negative diagonal minor in $\mathbf{V}(\mathbf{j})$.

By analyzing the negative terms, in $\alpha_{3}-\alpha_{6}$ and the diagonal minors of $\mathbf{V}(\mathbf{j})$, we have identified the origin of possible instabilities: four distinct destabilizing feedback cycles (I, II, III, and IV) are responsible for the existence

\begin{tabular}{|c|c|c|c|c|}
\hline Cycle & I & II & III & IV \\
\hline $\mathrm{Q}$ & & + & + & \\
\hline $\mathrm{H}_{2} \mathrm{Q}$ & + & & & + \\
\hline $\mathrm{SQ}^{-{ }^{-}}$ & & & & + \\
\hline $\mathrm{O}_{2}$ & & + & & + \\
\hline $\mathrm{O}_{2}^{--}$ & & & + & \\
\hline MB & + & & & + \\
\hline LMB & & + & + & \\
\hline $\mathrm{MB}^{\cdot}$ & + & + & + & \\
\hline$E_{i}$ & $\begin{array}{c}\left(E_{7}\right), \\
\left(E_{8}\right)\end{array}$ & $\left(\begin{array}{lll}E_{2} & E_{4} & E_{7}\end{array}\right)$ & $\left(E_{4} E_{7}\right)$ & $\begin{array}{lll}\left(\begin{array}{lll}E_{4} & E_{6} & E_{7}\end{array}\right), \\
\left(\begin{array}{lll}E_{4} & E_{6} & E_{8}\end{array}\right)\end{array}$ \\
\hline
\end{tabular}
of unstable steady states with the corresponding dynamic variables as summarized in Table II.

\section{A. Dominant destabilizing feedback cycle}

Although all four cycles can, in principle, produce unstable steady states, the simplest cycle with the most flexible instability conditions is the most important because this is expected to be the experimentally most accessible.
TABLE II. Destabilizing cycles with their relevant species and the corresponding minimal sub-networks

To identify this dominant cycle, we have compared them in terms of the numbers of interacting species and the size of the minimal sub-network required for their existence (see Table II).

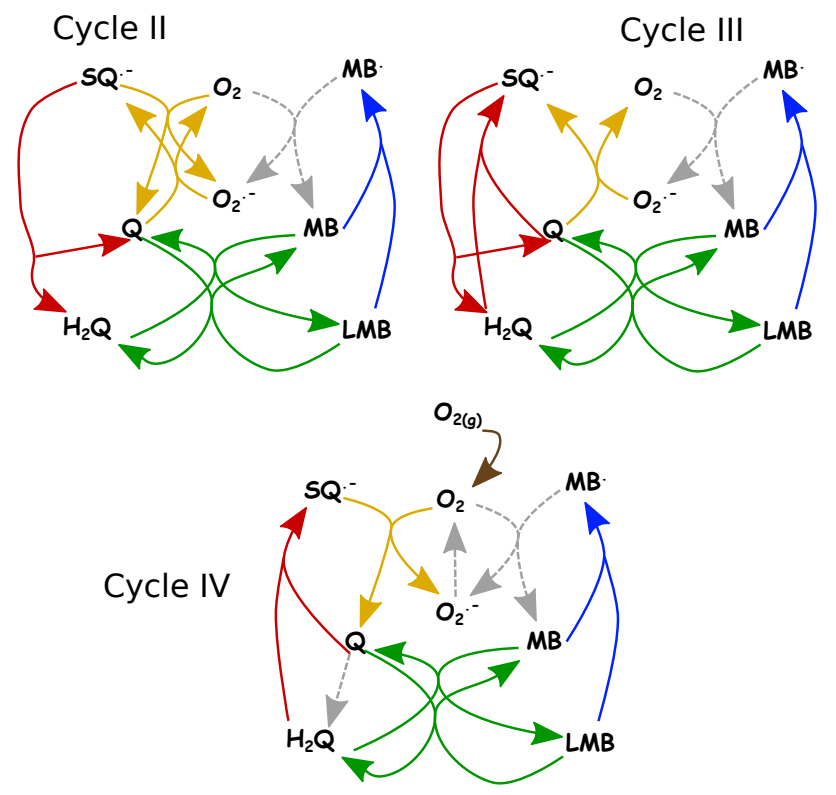

FIG. 3. Schematic representation of destabilizing cycles II, III and IV. Diagrams represents minimal set of columns of E required for cycle to be unstable; Cycle II $\left(E_{2} E_{4} E_{7}\right)$, Cycle III $\left(E_{4} E_{7}\right)$, Cycle IV $\left(E_{4} E_{6} E_{8}\right)$

Analysis has shown that cycle I is the dominant because this cycle is composed of only three interacting species, unlike the rest. Furthermore, cycles II-IV include at least one species from cycle I. For cycle I to become unstable, it is necessary to include only a subnetwork represented by extreme currents of $E_{7}$ or $E_{8}$. For cycles II-IV, summarized in Fig. 3, to become unstable, two or more columns of matrix $\mathbf{E}$ have to be combined but all unstable combinations contain $E_{7}$ or $E_{8}$. Since extreme currents of $E_{7}$ and $E_{8}$ are present in all destabilizing cycles, the coupling between $\mathrm{Q}$ and $\mathrm{MB}$ redox 
cycles is crucial for the appearance of instabilities. This conclusion is also supported by the fact that sub-network $E_{6}$, which incorporates only redox cycle related to $\mathrm{Q}$ in the presence of $\mathrm{O}_{2}$, cannot become unstable unless $E_{7}$ or $E_{8}$ is present.

Two types of instability are identified within the network: saddle-node bifurcation where bistability is sought and Andronov-Hopf-bifurcation which represents the birth of oscillatory dynamics. These instabilities are addressed in the next section.

\section{B. Derivation of the conditions for Andronov-Hopf and saddle-node bifurcations}

The large number of parameters $\left(j_{1}-j_{8}\right.$ and $\left.h_{1}-h_{8}\right)$ that determine the stability of the model complicates the derivation of the exact conditions for bifurcations, therefore the analysis has to be conducted in several steps. In the first step, conditions under which cycle I becomes unstable is determined by analyzing the negative diagonal minor of matrix $\mathbf{V}(\mathbf{j})$ of cycle $\mathrm{I}$. This results in the inequality

$\left(j_{1}+j_{6}\right)\left[2 j_{3} j_{4}+\left(j_{3}+j_{4}\right) J_{7,8}\right]<J_{7,8}\left(j_{3}+J_{7,8}\right)\left(j_{4}+J_{7,8}\right)$

where $J_{7,8}=j_{7}+j_{8}$. Parameters $j_{1}$ and $j_{6}$ can only be found on the left side of this inequality and therefore they represent the main stabilizing terms. Contrarily, $J_{7,8}$ is essential for the existence of instabilities and therefore the sum of $j_{7}$ and $j_{8}$ is the main destabilizing term. Inequality (8) can be satisfied for various current rates, but setting all $j_{i}$ parameters to have the same value is the most suitable choice for several reasons. Firstly, when all current rates are equal, cycles II-IV are stable. Secondly, this choice leads to a reduction in the number of parameters: there is only one current rate assigned as $j_{0}$. Moreover, approximate analytical expressions for the steady-state concentrations as a function of rate constants can be calculated from eqn (5). Lastly, setting $j_{1}-j_{8}$ to have the same value as $j_{0}$ leads to simplification in the expressions for $\alpha_{3}-\alpha_{6}$ (all of them still have negative terms) which allows us to derive conditions for the appearance of $\mathrm{AH}$ and $\mathrm{SN}$ bifurcations.

For SN bifurcation to emerge, it is necessary that $\alpha_{6}=0$. By calculating $\alpha_{6}$ and solving for $h_{7}$ conditions (A1) and (A2) listed in Appendix A are derived. Although $\mathrm{O}_{2}$ has to be present in the system for SN bifurcation to occur, its steady-state concentration does not determine the condition for appearance of SN bifurcation directly, as seen from eqn (A1) in Appendix A. Condition for SN bifurcation is determined by delicate balances between concentrations of $\mathrm{Q}$ and $\mathrm{MB}$ species. Condition (A2) in Appendix A ensures that only nonnegative values of $h_{7}$ are considered and it also shows that the critical value of $h_{2}$ is determined only by the values of $h_{1}$ and $h_{3}$, i.e., by the total amount of quinone in the system. From eqn (A2) it follows that $h_{2}$ has to be greater than $h_{1}$ and $h_{3}$. Since $h_{1}, h_{2}$, and $h_{3}$ correspond to the reciprocal steady-state concentrations of $\mathrm{Q}$, $\mathrm{H}_{2} \mathrm{Q}$ and $\mathrm{SQ}^{-}$, respectively, for $\mathrm{SN}$ bifurcation to take place the steady-state concentration of $\mathrm{H}_{2} \mathrm{Q}$ has to be low compared to that of $\mathrm{Q}$ and $\mathrm{SQ}^{-}$according to

$$
\left[\mathrm{H}_{2} \mathrm{Q}\right]_{s s}<\frac{5}{182}\left[\mathrm{SQ}^{--}\right]_{s s}+\frac{54}{182}[\mathrm{Q}]_{s s},
$$

the transformed form of eqn (A2). Analysis of eqn (A1) also reveals that $h_{7}$ has to be less than $h_{6}$ and $h_{8}$. Seeing that $h_{6}, h_{7}$, and $h_{8}$ represent the reciprocal steady-state concentrations of $\mathrm{MB}, \mathrm{LMB}$, and $\mathrm{MB}$, respectively, $\mathrm{SN}$ bifurcation will appear when LMB steady-state concentration is greater than that of $\mathrm{MB}$ and $\mathrm{MB}^{\circ}$. By using eqn (A2) in Appendix A, it is possible to determine the position of SN bifurcation as a function of these concentrations, as shown in Fig. 4. For instability to arise the methylene blue redox system has to be in its reduced state with LMB dominating over the other oxidized forms in the steady state (see Figs. 4(c,d)). Comparison of these two figures also reveals that the reactive $\mathrm{MB}^{\cdot} \mathrm{rad}-$ ical is expected to be present in concentrations significantly smaller than the others in accordance with chemical intuition. Large $[\mathrm{LMB}] /[\mathrm{Q}]$ ratio favors stable steady state under pool conditions, therefore an increase in the concentration of $\mathrm{Q}$ destabilizes the steady state while the concentration of the reactive semiquinone radical remains small (see Figs. 4(a,b)).
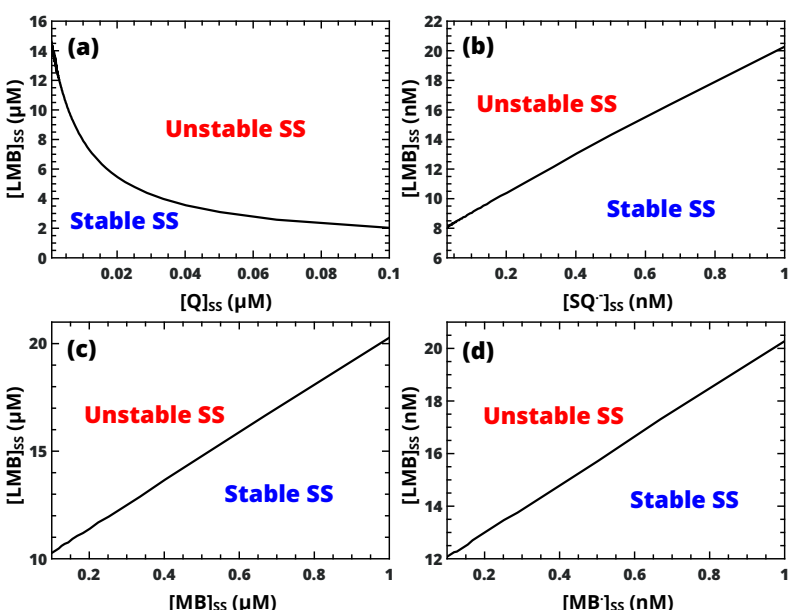

FIG. 4. Location of saddle-node bifurcation in the concentration space with the value of $h_{2}$ set to be twice the critical value defined by eqn (A2) in Appendix A.

For the emergence of Andronov-Hopf bifurcation conditions (B1) and (B2), displayed in Appendix B, are derived from coefficient $\alpha_{5}$. Inequality (B1) is an approximation of the exact condition for the emergence of $\mathrm{AH}$ bifurcation but it has proved to be very reliable. The values of $h_{4}$ and $h_{7}$, i.e., the steady-state concentrations of $\mathrm{O}_{2}$ and LMB, are found to be crucial. Condition (B2) have to be satisfied in order for $h_{4}$ to have non-negative 
values. By using eqn (B1), the position of AH bifurcation as a function of $h_{4}$ and $h_{1}, h_{2}, h_{6}$ and $h_{8}$ are examined for the required lower value of $h_{7}$, i.e., higher concentration of LMB. Figures 5(a,b) show that the hydroquinone/quinone system has to be in the oxidized state because $[\mathrm{Q}]_{s s}>\left[\mathrm{H}_{2} \mathrm{Q}\right]_{s s}$ is needed for the instability to arise. Low concentration of MB also favors the instability (see Fig. 5(c)), while the reactive $\mathrm{MB}^{*}$ remains in low concentration as expected for chemically realistic conditions (Fig. 5(d)).
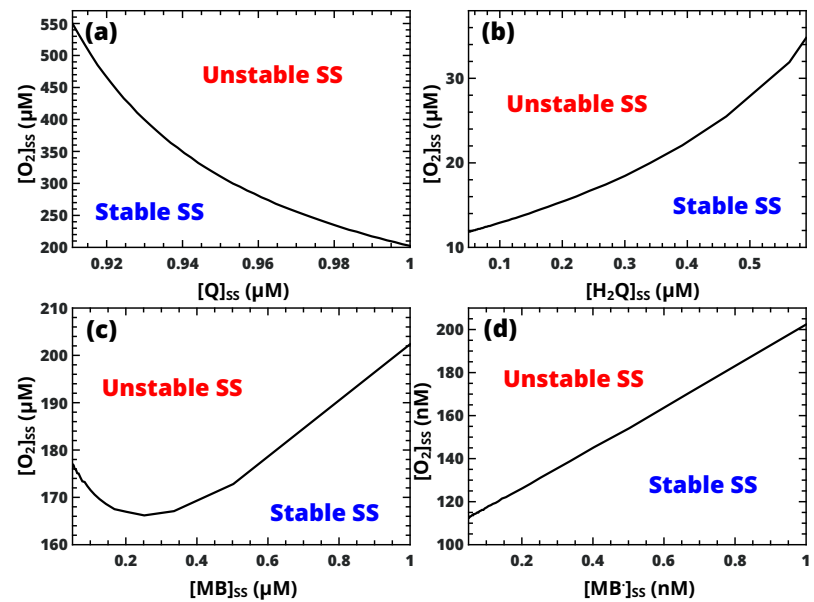

FIG. 5. Location of Andronov-Hopf bifurcation in the concentration space with the value of $h_{7}$ set to be one fifth of the critical value defined by eqn (B2) in Appendix B.

\section{Numerical bifurcation analysis}

The conditions for $\mathrm{AH}$ and $\mathrm{SN}$ bifurcations discussed in the previous section have proved to be very accurate but they are the functions of numerous $h_{i}$ parameters. Therefore, an additional investigation has been carried out in order to further confirm their validity and more importantly to test the significance of parameters for the emergence of $\mathrm{AH}$ and $\mathrm{SN}$ bifurcations. The numerical bifurcation analysis is performed by setting the values of $j_{0}$ and $h_{1}-h_{8}$ to 1 , then one or a combination of several $h_{i}$ parameters are varied while all other are kept constant. In each step, selected $h_{i}$ parameters are given the same value, the Hurwitz determinants are numerically calculated, and the number of sign changes in the Routh-Hurwitz (RH) array are evaluated. In this way, the parameters important for the emergence of $\mathrm{AH}$ and SN bifurcations can be detected.

There are two essential combinations $\left(h_{1}, h_{7}\right)$ and $\left(h_{4}\right.$, $h_{7}$ ) that lead to the appearance of AH and SN bifurcations. All of them include $h_{7}$, which suggests that appropriate steady-state concentration of LMB is essential for the emergence of unstable steady states. On the one hand, the decrease in $h_{1}$ and $h_{7}$ leads to the appearance of a single sign change in the Routh-Hurwitz array, corresponding to the loss of stability via saddle-node bifurcation. This is in accordance with the phase diagram in Fig. 4(a), where high steady-state concentrations of LMB and $\mathrm{Q}$ favor instability and confirm the conditions in eqn (A1)-(A2) of Appendix A. On the other hand, upon decreasing the $\left(h_{4}, h_{7}\right)$ pair, a double sign change in the Routh-Hurwitz array occurs, indicating the loss of stability via Andronov-Hopf bifurcation. This means that high steady-state concentrations of LMB and $\mathrm{O}_{2}$ are essential for the existence of oscillatory dynamics, which is also in agreement with the conditions in eqn (B1)-(B2) of Appendix B and further confirms the validity of eqn (A1)-(B2).

Up to this point, sodium borohydride is considered as a pool chemical with constant concentration. When we inspect the stability of the steady state as a function of borohydride concentration, we find stable steady states at low concentration, however upon increasing the amount of borohydride, a bistable region bounded by saddle-node bifurcations (Fig. 6) or an oscillatory region between Andronov-Hopf bifurcations (Fig. 7) can be found, depending on the parameters. Greater reducing agent concentrations are again characterized with stable steady states.

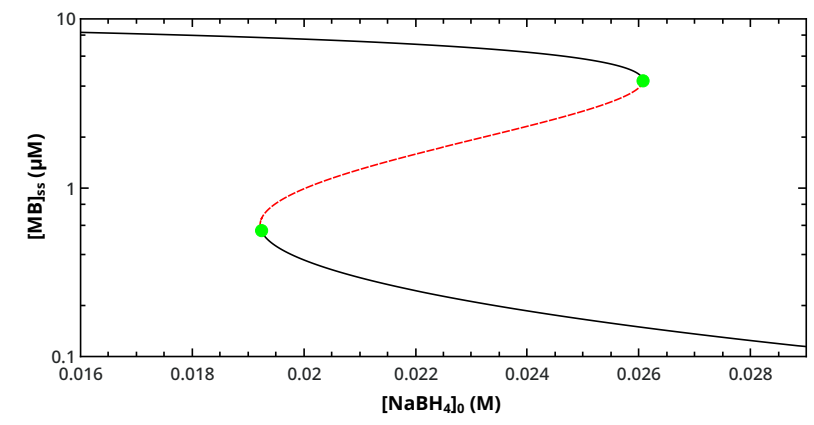

FIG. 6. Bistable region bounded by oxidized and reduced states as the sodium borohydride initial concentration is increased.

Considering that oxygen is also present in the system, the delicate balance between the oxydizing power of reactive oxygen species and the reducing power of borohydride can lead to the instability giving rise to bistability or oscillations. At low borohydride concentration, oxidation driven by ROS dominates, oppositely to high concentration where the fast reduction, both of which are present in reaction routes $E_{6}$ and $E_{8}$. These routes themselves cannot destabilize the steady state, for that $E_{4}$ is needed in cycles II-IV, i.e. the coupling between the two redox systems, the quinone and the methylene blue in this particular model.

In a closed system with initial conditions matching the bistable region in the corresponding pool chemical system, reaction paths with fast and slow time scales representing an autocatalytic burst are feasible. With parameters placing the initial conditions in the range be- 


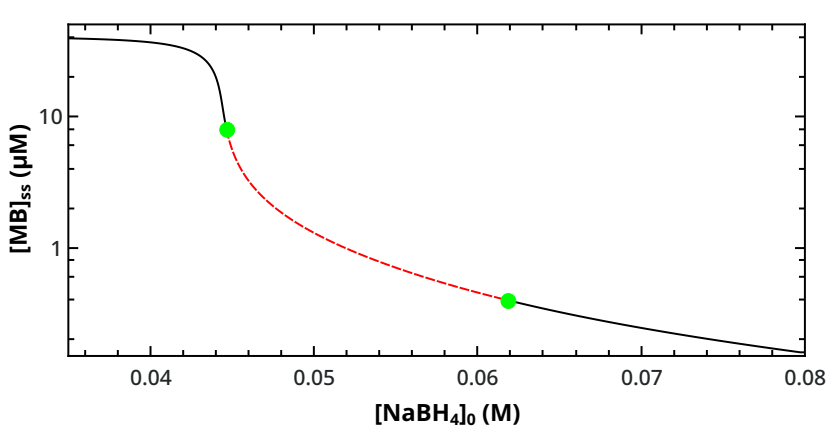

FIG. 7. Two Andronov-Hopf bifurcations borders the region with unstable steady state that allow oscillations for a range of sodium borohydride initial concentration.

tween the Andronov-Hopf bifurcations, transient oscillation can be achieved as shown in Fig. 8.

The original parameters used by Taran et al. ${ }^{29}$ do not comprise conditions in the vicinity of an AndronovHopf bifurcation, no oscillations have been observed with them, therefore a systematic search within the parameter space is required to locate the desired behavior. One
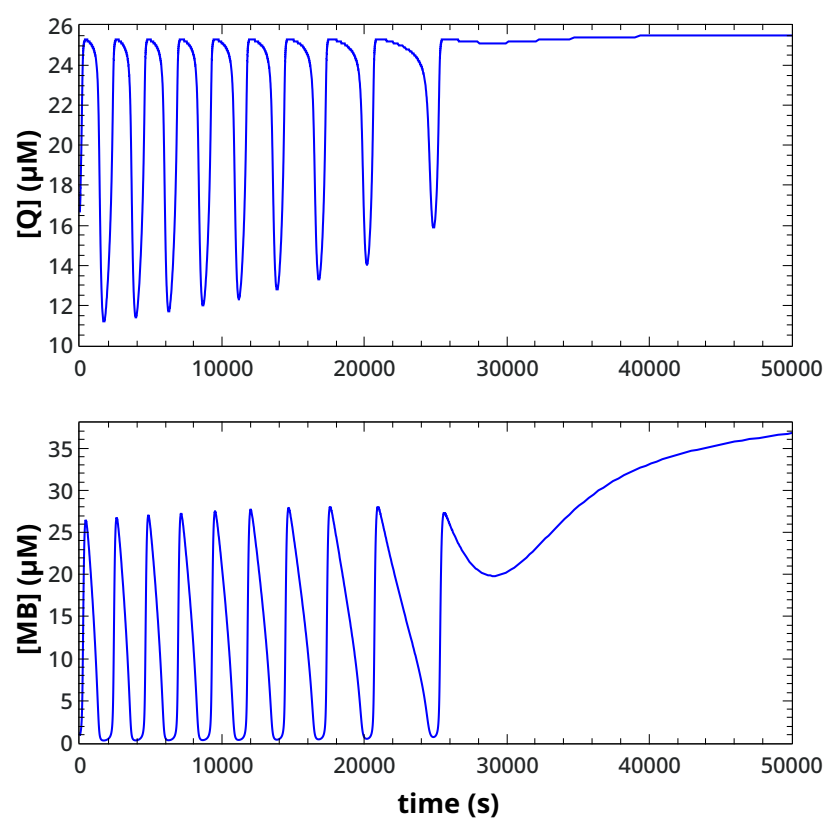

FIG. 8. Numerical simulation obtained with adjusted parameters presented in Table I under batch conditions; Initial conditions used in simulation: $\left[\mathrm{NaBH}_{4}\right]_{0}=5 \times 10^{-2} \mathrm{M}$, $[\mathrm{Q}]_{0}=2.58 \times 10^{-5} \mathrm{M},[\mathrm{MB}]_{0}=4.10 \times 10^{-5} \mathrm{M}$. Initial concentrations of remaining species were set to 0 .

method to find the required parameter set is to fix all $j_{i}$ current rates to the same value of $j_{0}$, which results in a simplified version of eqn (5). Using the relation between $r_{i, s s}$ and $j_{0}$, analytical expressions for the steady-state concentrations as functions of reaction rates $k_{i}$ can be derived. There are 13 relations in eqn (C1) of Appendix $\mathrm{C}$ that need to be satisfied but only 8 species and one $j_{0}$ parameter, therefore four rate constants are expressed as a function of the remaining. The value of $j_{0}$ is determined only by the value of $k_{8}$ which represents the rate at which $\mathrm{O}_{2}$ enters the system. Since $j_{0}$ is involved in all steady-state relations, this rate governs the dynamics which is in agreement with the results of stability and bifurcation analysis, since it appears in both $E_{6}$ and $E_{8}$.

Detailed investigation has shown that the condition in eqn (B1) cannot be satisfied by changing only the reactant concentrations in the parameter set of Taran et al. ${ }^{29}$ In other words, modified rate constants are necessary, which would require the change of reducing agent, electron acceptor or even a different hydroquinone may be needed for the experimental realization. One set of adjusted rate constants that match eqn (B1) is presented in Table I. During the parameter search we have fixed the rate constant of (R7) because the ROS dynamics associated with it does not change. The general outcome can be summarized as the following. The values of $k_{3}$ and $k_{-3}$ have to be reduced, however the relation $k_{3}>k_{-3}$ can be preserved in accordance with the original set of values. For oscillatory dynamics steady-states with the electron acceptor in its reduced state, i.e. high LMB concentrations, are needed, therefore the values of $k_{6}$ and $k_{-6}$ have been changed significantly such that the former has become greater. Direct numerical simulation carried out with one set of adjusted rate constants under batch conditions produces temporal oscillation with appropriate initial conditions (see Fig. 8). Although these rate constants are chemically realistic, for constructing a specific experiment to observe this oscillatory dynamics, a different electron acceptor has to be selected instead of methylene blue where the equilibrium constant between the redox pairs match the necessary conditions.

\section{CONCLUSION}

Detailed analysis of the model describing the dynamics of ROS in the rhizosphere has been conducted to explore its dynamical properties. Stability analysis has proved the existence of Andronov-Hopf and saddle-node bifurcations under appropriate conditions. Four destabilizing feedback loops govern the dynamics. The comprehensive analysis has also shown that the cycle involving hydroquinone $\left(\mathrm{H}_{2} \mathrm{Q}\right)$, the electron acceptor $(\mathrm{MB})$ and its semi-reduced form $\left(\mathrm{MB}^{*}\right)$ itself is dominant because it is responsible for the emergence of bifurcations. The conditions for the appearance of bifurcations are also derived and their validity has been corroborated by numerical analysis.

We have identified that higher steady-state concentrations of the reduced electron acceptor (LMB) are required for obtaining unstable steady states. The type of instabilities are then determined by the steady-state concen- 
tration of the dissolved oxygen: at low concentrations saddle-node bifurcations may emerge, while high steadystate concentration is required for oscillatory dynamics. In both cases, preserving the delicate balance between the hydroquinon/quinone system and the electron acceptor is essential.

By selecting methylene blue as the electron acceptor to couple with the hydroquinone/quinone system and sodium borohydride as the reducing agent in excess, ${ }^{29}$ bursts can be produced that lead to reaction-diffusion fronts with the experimentally accessible parameter set. In the rizosphere the ROS-hydroquinone reaction network, as we have shown, can have the capability of yielding oscillatory dynamics that can drive biologically important processes depending on the electron acceptor and the reducing species. Nonlinear responses, such as autocatalytic bursts and oscillations, can then provide the regulatory signals that are essential in living systems.

\section{DATA AVAILABILITY STATEMENT}

The data that support the findings of this study are available from the corresponding author upon reasonable request.

\section{ACKNOWLEDGMENTS}

This work was supported by the National Research, Development and Innovation Office (K119795) and GINOP-2.3.2-15-2016-00013 project. S.M. acknowledges the support by the Ministry of Education, Sciences and Technology of the Republic of Serbia (172015 and 45103-9/2021-14/200146).

\section{Appendix A: Condition for saddle-node bifurcation}

Regarding the reciprocal steady-state concentrations $\left(h_{i}\right)$ in the stoichiometric network analysis, the inequality

$$
h_{7} \leq \frac{3 h_{6} h_{8}\left(5 h_{1} h_{2}-182 h_{1} h_{3}+54 h_{2} h_{3}\right)}{540 H_{126}+72 h_{3} h_{6}\left(h_{2}+17 h_{1}\right)+607 H_{128}+734 H_{138}+258 H_{238}}
$$

with $H_{i j k}=h_{i} h_{j} h_{k}$ must be satisfied for saddle-node bifurcation to occur, where the right hand side must be positive. This results in the additional requirement of

$$
h_{2}>\frac{182 h_{1} h_{3}}{5 h_{1}+54 h_{3}} .
$$

\section{Appendix B: Condition for Andronov-Hopf bifurcation}

Regarding the reciprocal steady-state concentrations $\left(h_{i}\right)$ in the stoichiometric network analysis, the inequality

$$
h_{4}<\frac{-2 h_{5}\left(3 H_{1267}-2 H_{1268}+6 H_{1367}+5 H_{1278}+6 H_{1378}-4 H_{2368}+4 H_{2378}\right)}{h_{5}\left(6 H_{126}+5 H_{127}+8 H_{136}+6 H_{137}+4 H_{236}+4 H_{237}+6 H_{167}+3 H_{267}+12 H_{367}\right)+3 h_{6}\left(H_{127}+2 H_{137}\right)}
$$

must be satisfied for Andronov-Hopf bifurcation to occur, where $H_{i j k}=h_{i} h_{j} h_{k}$ and $H_{i j k l}=h_{i} h_{j} h_{k} h_{l}$. This is accompanied with the additional requirement of

$$
h_{7}<\frac{2 H_{268}\left(h_{1}+2 h_{3}\right)}{3 h_{1} h_{6}\left(h_{2}+2 h_{3}\right)+5 H_{128}+2 h_{3} h_{8}\left(3 h_{3}+2 h_{2}\right)}
$$

\section{Appendix C: Steady states as a function of rate constants}

The dependence of steady-state concentrations on the rate constant is provided by

$$
\begin{array}{lll}
{[\mathrm{Q}]=\frac{2 k_{8}}{3 k_{1}},} & {\left[\mathrm{H}_{2} \mathrm{Q}\right]=\frac{k_{1}}{k_{2}}, \quad\left[\mathrm{SQ}^{--}\right]=\frac{1}{3} \sqrt{\frac{6 k_{8}}{k_{-2}}},[\mathrm{MB}]=\frac{k_{2} k_{8}}{k_{1} k_{6}}} \\
{[\mathrm{LMB}]=\frac{k_{1}}{2 k_{-6}},} & {\left[\mathrm{MB}^{\cdot}\right]=\sqrt{\frac{k_{8}}{3 k_{-4}}},\left[\mathrm{O}_{2}\right]=\frac{k_{8}}{3 k_{-8}}, \quad\left[\mathrm{O}_{2}^{--}\right]=\frac{1}{3} \sqrt{\frac{6 k_{8}}{k_{7}}}}
\end{array}
$$


along with

$$
j_{0}=\frac{k_{8}}{3}, k_{3}=\frac{3 k_{-8}}{2} \sqrt{\frac{6 k_{-2}}{k_{8}}}, k_{-3}=\frac{3 k_{1}}{4} \sqrt{\frac{6 k_{7}}{k_{8}}}, k_{5}=4 k_{-8} \sqrt{\frac{3 k_{-4}}{k_{8}}}, k_{6}=\frac{k_{2} k_{4}}{2 k_{-6}}
$$

This allows the identification of parameter sets that match the desired behavior.

${ }^{1}$ I. R. Epstein and J. A. Pojman, An Introduction to Nonlinear Dynamics: Oscillations, Waves, Patterns, and Chaos (Oxford University Press, Oxford, 1998).

${ }^{2}$ G. Hu, J. A. Pojman, S. K. Scott, M. M. Wrobel, and A. F. Taylor, "Base-catalyzed feedback in the urea- urease reaction," J. Phys. Chem. B 114, 14059-14063 (2010).

${ }^{3}$ N. E. Kouvaris, M. Sebek, A. S. Mikhailov, and I. Z. Kiss, "Selforganized stationary patterns in networks of bistable chemical reactions," Angew. Chem. Int. Ed. 55, 13267-13270 (2016).

${ }^{4}$ I. Maity, N. Wagner, R. Mukherjee, D. Dev, E. Peacock-Lopez, R. Cohen-Luria, and G. Ashkenasy, "A chemically fueled nonenzymatic bistable network," Nature Comm. 10, 4636 (2019).

${ }^{5}$ A. Zlotnik, R. Nagao, I. Z. Kiss, and J.-S. Li, "Phase-selective entrainment of nonlinear oscillator ensembles," Nature Comm. 7, 10788 (2016)

${ }^{6}$ S. N. Semenov, L. J. Kraft, A. Ainla, M. Zhao, M. Baghbanzadeh, V. E. Campbell, K. Kang, J. M. Fox, and G. M. Whitesides, "Autocatalytic, bistable, oscillatory networks of biologically relevant organic reactions," Nature 537, 656-660 (2016).

${ }^{7}$ J. Leira-Iglesias, A. Tassoni, T. Adachi, M. Stich, and T. M. Hermans, "Oscillations, travelling fronts and patterns in a supramolecular system," Nature Nanotech. 13, 1021-1027 (2018).

${ }^{8}$ B. Bohner, T. Bánsági, A. Tóth, D. Horváth, and A. F. Taylor, "Periodic nucleation of calcium phosphate in a stirred biocatalytic reaction," Angew. Chem. Int. Ed. , 2823-2828 (2020). ${ }^{9}$ I. Z. Kiss, W. Wang, and J. L. Hudson, "Complexity of globally coupled chaotic electrochemical oscillators," Phys. Chem. Chem. Phys. 2, 3847-3854 (2000).

${ }^{10}$ M. A. Budroni, I. Calabrese, Y. Miele, M. Rustici, N. Marchettini, and F. Rossi, "Control of chemical chaos through medium viscosity in a batch ferroin-catalysed belousov-zhabotinsky reaction," Phys. Chem. Chem. Phys. 19, 32235-32241 (2017).

${ }^{11}$ G. Bodó, R. M. Branca, Á. Tóth, D. Horváth, and C. Bagyinka, "Concentration-dependent front velocity of the autocatalytic hydrogenase reaction," Biophys. J. 96, 4976-4983 (2009)

${ }^{12}$ D. G. Míguez, V. K. Vanag, and I. R. Epstein, "Fronts and pulses in an enzymatic reaction catalyzed by glucose oxidase," Proc. Natl. Acad. Sci. USA 104, 6992-6997 (2007)

${ }^{13}$ M. M. Wrobel, T. Bánsági Jr, S. K. Scott, A. F. Taylor, C. O. Bounds, A. Carranza, and J. A. Pojman, "ph wave-front propagation in the urea-urease reaction," Biophys. J. 103, 610-615 (2012).

14.J. Sneyd, J. M. Han, L. Wang, J. Chen, X. Yang, A. Tanimura, M. J. Sanderson, V. Kirk, and D. I. Yule, "On the dynamical structure of calcium oscillations," Proc. Natl. Acad. Sci. USA 114, 1456-1461 (2017).

${ }^{15}$ R. Balaji, C. Bielmeier, H. Harz, J. Bates, C. Stadler, A. Hildebrand, and A.-K. Classen, "Calcium spikes, waves and oscillations in a large, patterned epithelial tissue," Sci. Rep. 7, 42786 (2017).

16 A.-K. Gustavsson, C. B. Adiels, B. Mehlig, and M. Gokso, "Entrainment of heterogeneous glycolytic oscillations in single cells," Sci. Rep. 5, 9404 (2015).

${ }^{17}$ A. Weber, W. Zuschratter, and M. J. B. Hauser, "Partial synchronisation of glycolytic oscillations in yeast cell populations," Sci. Rep. 10, 19714 (2020).

${ }^{18}$ J.-C. Leloup and A. Goldbeter, "Toward a detailed computational model for themammalian circadian clock," Proc. Natl. Acad. Sci. USA 100, 7051-7056 (2003).

19 J. D. Veldhuis, D. M. Keenan, and S. M. Pincus, "Motivations and methods for analyzing pulsatile hormone secretion," Endocr. Rev. 29, 823-864 (2008)

${ }^{20}$ K. L. Gamble, R. Berry, S. J. Frank, and M. E. Young, "Circadian clock control of endocrine factors," Nat. Rev. Endocrinol. 10, 466-475 (2014)

${ }^{21}$ V. M. Marković, Ž. Čupić, S. Maćešić, A. Stanojević, V. Vukojević, and L. Kolar-Anić, "Modelling cholesterol effects on the dynamics of the hypothalamic-pituitary-adrenal (hpa) axis," Math. Med. Biol. 33, 1-28 (2014).

${ }^{22}$ G. B. Monshausen, T. Bibikova, M. Messerli, C. Shi, and S. Gilroy, "Oscillations in extracellular ph and reactive oxygen species modulate tip growth of arabidopsis root hairs," Proc. Natl. Acad. Sci. USA 104, 20996-21001 (2007).

${ }^{23}$ Z. A. Wood, L. B. Poole, and P. A. Karplus, "Peroxiredoxin evolution and the regulation of hydrogen peroxide signaling," Science 300, 650-653 (2003)

${ }^{24}$ M. Potockỳ, M. A. Jones, R. Bezvoda, N. Smirnoff, and V. Žárskỳ, "Reactive oxygen species produced by nadph oxidase are involved in pollen tube growth," New Phytol. 174, 742-751 (2007).

${ }^{25}$ S. S. Gill and N. Tuteja, "Reactive oxygen species and antioxidant machinery in abiotic stress tolerance in crop plants," Plant physiology and biochemistry 48, 909-930 (2010).

${ }^{26} \mathrm{C}$. H. Foyer and G. Noctor, "Redox homeostasis and antioxidant signaling: a metabolic interface between stress perception and physiological responses," Plant Cell 17, 1866-1875 (2005).

${ }^{27} \mathrm{~B}$. Halliwell, "Reactive species and antioxidants. redox biology is a fundamental theme of aerobic life," Plant Physiol. 141, 312$322(2006)$

${ }^{28}$ A. Krieger-Liszkay, C. Fufezan, and A. Trebst, "Singlet oxygen production in photosystem ii and related protection mechanism," Photosynthesis Research 98, 551-564 (2008).

${ }^{29}$ O. Taran, V. Patel, and D. G. Lynn, "Small molecule reaction networks that model the ros dynamics of the rhizosphere," Chem. Commun. 55, 3602-3605 (2019).

${ }^{30} \mathrm{Y}$. X. Zhang and R. J. Field, "Simplification of a mechanism of the methylene blue hydrosulfide-oxygen cstr oscillator: A homogeneous oscillatory mechanism with nonlinearities but no autocatalysis," J. Phys. Chem. 95, 723-727 (1991).

${ }^{31}$ B. L. Clarke, "Stability of complex reaction networks," Adv Chem. Phys. , 1-215 (1980).

${ }^{32}$ G. Schmitz, L. Z. Kolar-Anic, S. R. Anic, and Z. D. Cupic, "Stoichiometric network analysis and associated dimensionless kinetic equations. application to a model of the bray- liebhafsky reaction," J. Phys. Chem. A 112, 13452-13457 (2008).

${ }^{33}$ V. Radojkovič and I. Schreiber, "Constrained stoichiometric network analysis," Phys. Chem. Chem. Phys. 20, 9910-9921 (2018).

${ }^{34}$ J. A. C. Gallas, M. J. B. Hauser, and L. F. Olsen, "Complexity of a peroxidase-oxidase reaction model," Phys. Chem. Chem. Phys. 23, 1943-1955 (2021).

${ }^{35}$ M. A. Budroni, E. Biosa, S. Garroni, G. R. C. Mulas, N. Marchettini, N. Culeddu, and M. Rustici, "Understanding oscillatory phenomena in molecular hydrogen generation via sodium borohydride hydrolysis," Phys. Chem. Chem. Phys. 15, 18664-18670 (2013).

${ }^{36}$ M. A. Budroni, S. Garroni, G. Mulas, and M. Rustici, "Bursting dynamics in molecular hydrogen generation via sodium borohydride hydrolysis," J. Phys. Chem. C 121, 4891-4898 (2017).

${ }^{37}$ O. Hadač and I. Schreiber, "Stoichiometric network analysis of the photochemical processes in the mesopause region," Phys. Chem. Chem. Phys. 13, 1314-1322 (2011). 
${ }^{38}$ D. Hochberg, R. D. Bourdon Garcia, J. A. Agreda Bastidas, and J. M. Ribó, "Stoichiometric network analysis of spontaneousmirror symmetry breaking in chemical reactions," Phys. Chem. Chem. Phys. 19, 17618-17636 (2017).

${ }^{39}$ Ž. Čupić, S. Maćešić, K. Novakovic, S. Anić, and L. Kolar-Anić, "Stoichiometric network analysis of a reaction system with conservation constraints," Chaos 28, 083114 (2018).

${ }^{40}$ B. Von Hohenbalken, B. L. Clarke, and J. E. Lewis, "Least distance methods for the frame of homogeneous equation systems," J. Comput. Appl. Math. 19, 231-241 (1987).

${ }^{41}$ R. Schuster and S. Schuster, "Refined algorithm and computer program for calculating all non-negative fluxes admissible in steady states of biochemical reaction systems with or without some flux rates fixed," Bioinformatics 9, 79-85 (1993).
${ }^{42}$ P. E. Lehner and E. Noma, "A new solution to the problem of finding all numerical solutions to ordered metric structures," Psychometrika 45, 135-137 (1980).

${ }^{43}$ B. L. Clarke, "Stoichiometric network analysis," Cell Biophys. 12, 237-253 (1988).

${ }^{44}$ B. D. Aguda and B. L. Clarke, "Bistability in chemical reaction networks: theory and application to the peroxidase-oxidase reaction," J. Chem. Phys. 87, 3461-3470 (1987).

${ }^{45} \mathrm{~B}$. L. Clarke and W. Jiang, "Method for deriving hopf and saddlenode bifurcation hypersurfaces and application to a model of the belousov-zhabotinskii system," J. Chem. Phys. 99, 4464-4478 (1993). 
Accepted to J. Chem Ohys: 10.1063/5.0062139

2(g)

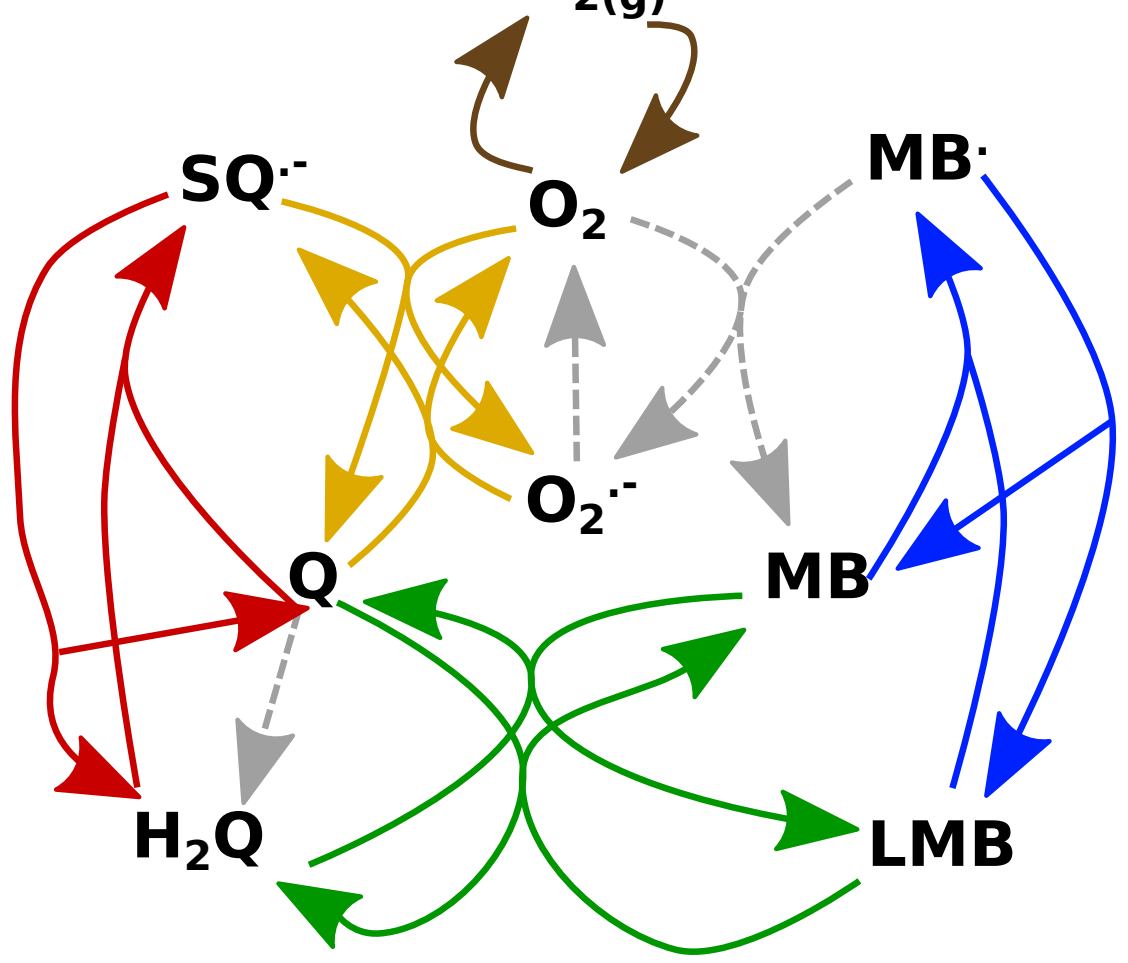


Cycle II
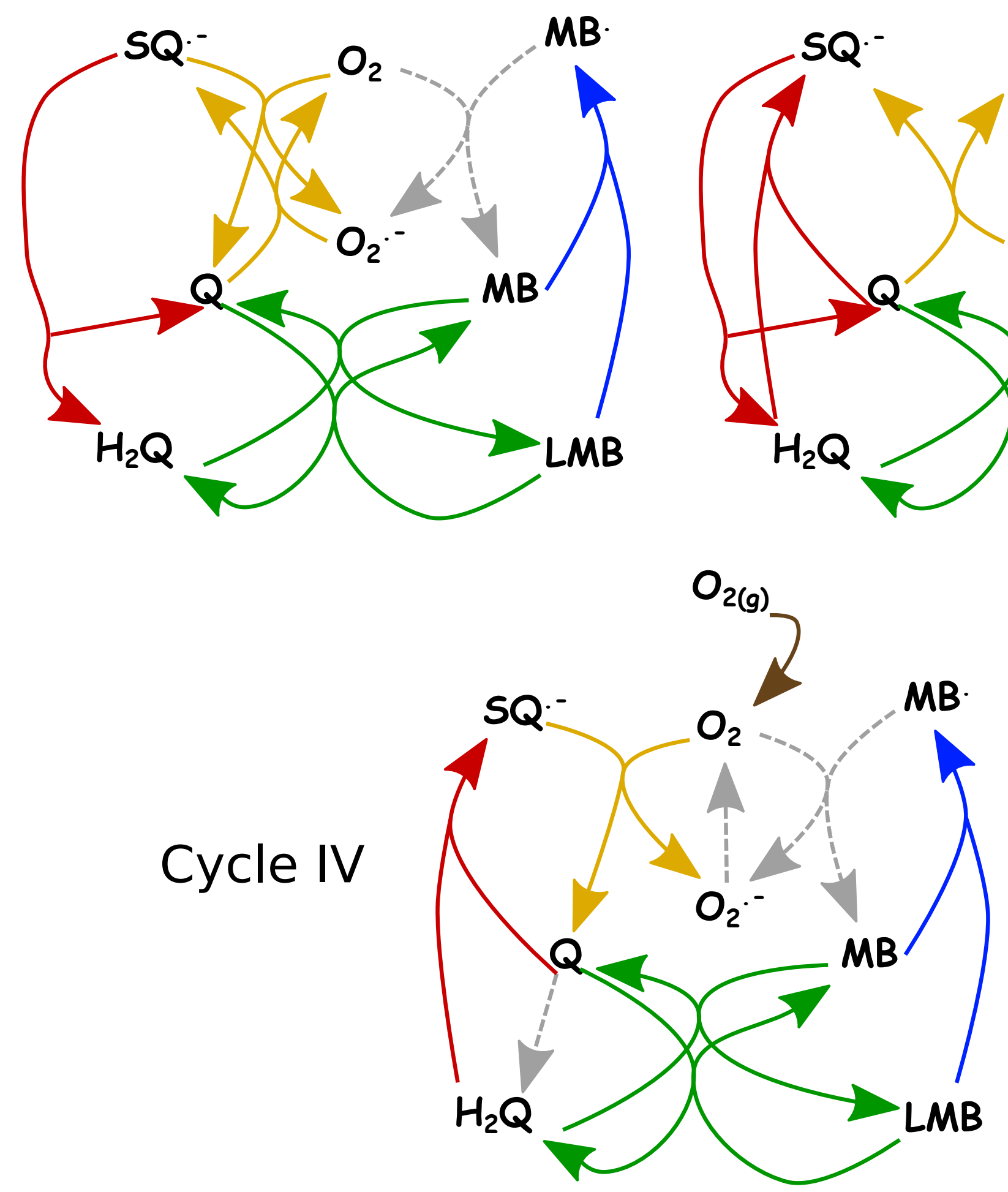


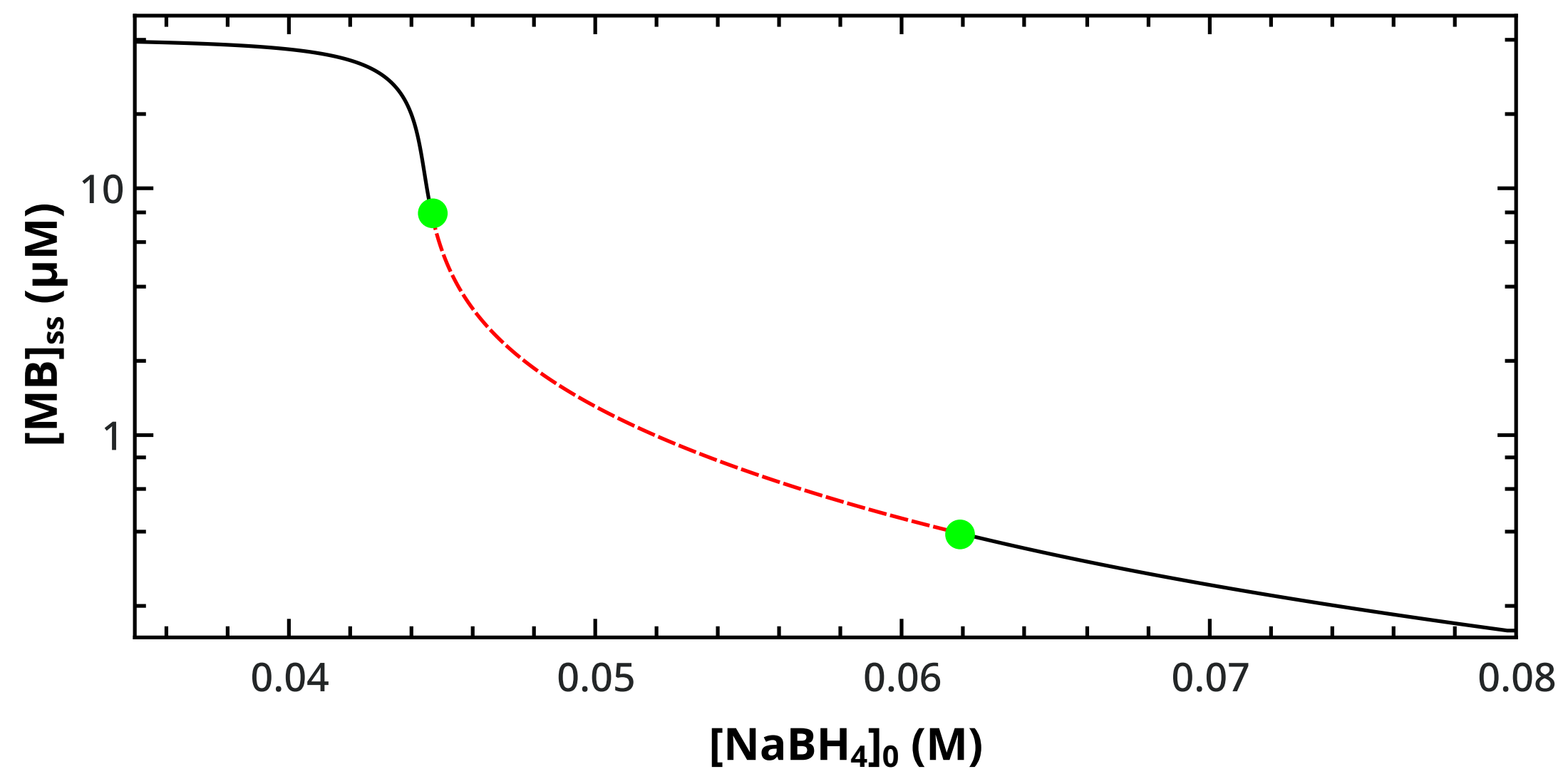



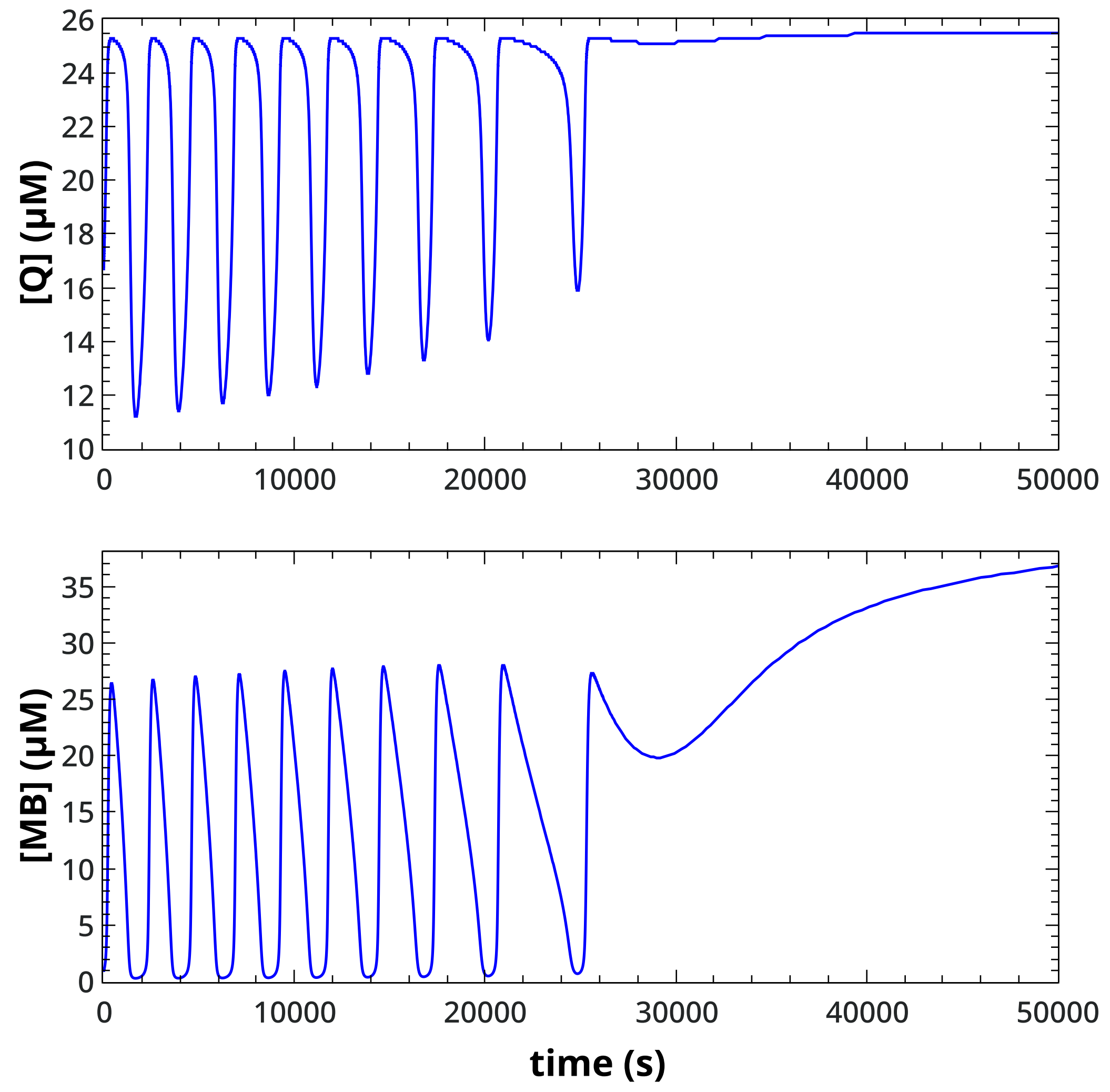